\title{
ESTUDO DA QUALIDADE DE VIDA EM PESSOAS COM LESÃO MEDULAR TRAUMÁTICA
}

\author{
Janaina Vall', Violante Augusta Batista Braga², Paulo César de Almeida ${ }^{3}$
}

\begin{abstract}
RESUMO - Centros de pesquisa do mundo inteiro vêm realizando estudos sobre qualidade de vida em diversas condições crônicas, com o intuito principal de promover intervenções que venham de encontro à sua melhoria. Este fato se estende aos pacientes portadores de lesão medular. Este é um estudo com o objetivo de avaliar a qualidade de vida das pessoas portadoras de lesão medular traumática, na cidade de Fortaleza, Ceará, Brasil. Participaram do estudo 32 pacientes paraplégicos adultos com lesão medular completa, de etiologia traumática, a maioria do sexo masculino, idade entre 20 a 47 anos e entre 5 a 10 anos de lesão. O instrumento utilizado para coleta dos dados foi o The MOS 36-item Short-Form Health Survey. Como resultado foi encontrado que o paciente com lesão medular traumática possui grande co $\mathrm{m}$ p rometimento de sua qualidade de vida, em todos os seus domínios, principalmente no que se re fe re aos "aspectos sociais".
\end{abstract}

PALAVRAS-CHAVE: paraplegia, qualidade de vida, reabilitação.

\begin{abstract}
Study of the quality of life in people with traumatic spinal cord injury
ABSTRACT - Centers of re se arch all over the world are accomplishing studies about quality of life in several ch ronic conditions, for promoting interventions that promote improvement. This fact extends to the spinal cord injury patients. This is a study with the aim of evaluating the quality of life of traumatic spinal cord injury people in the city of Fortaleza, Ceará, Brazil. Participated of the study 32 paraplegic adult patients with complete traumatic spinal cord injury; most were male, aged 20 to 47 years old and 5 to 10 years of lesion. The instrument used for collection of data was The MOS 36-item Short-Form Health Survey. As results we found that the traumatic spinal cord injury patient possesses great compromising of the quality of life, in all your domains, mainly in what it refers to the "social aspects".
\end{abstract}

KEY WORDS: paraplegia, quality of life, rehabilitation.

As lesões medulares são cada vez mais freqüentes devido principalmente ao aumento da violência urbana. Dentre as causas, o acidente de trânsito e a agressão por arma de fogo são as mais comuns. Os pacientes acometidos, em sua maioria, são jovens, do sexo masculino, solteiros e residentes em áreas urbanas ${ }^{1}$. Tais lesões geram uma incapacidade de alto custo para o governo e acarreta importantes alterações no estilo de vida do paciente. Ela causa perda parcial ou total da motricidade e sensibilidade, além de comprometimento vasomotor, intestinal, vesical e sexual ${ }^{2}$. Além de sua gravidade e irreversibilidade, exigem um programa de reabilitação longo e oneroso, que na maioria das vezes não leva à cura, mas à adaptação do indivíduo à sua nova condição. Esse p rocesso de reabilitação, no entanto, vai para além da prevenção dos danos causados pela lesão e obje- tiva principalmente melhora da qualidade de vida através da independência funcional, melhora da autoestima e inclusão social desses pacientes ${ }^{3}$.

Qualidade de vida é uma expressão de natureza muito debatida entre pesquisadores de diferentes áreas e ocupa cada vez mais espaço na política da saúde e da população. Mesmo assim, não se consegue ter uma definição ou conceito universal. Alguns autores defendem a teoria de que todas as pessoas tem um plano para sua vida e a qualidade de vida seria a diferença entre as esperanças e expectativas do indivíduo e a realidade presente; quanto menor esta diferença, melhor a qualidade de vida ${ }^{4}$. Segundo a O rganização Mundial de Saúde (OMS), qualidade de vida é a percepção do indivíduo em relação a sua posição na vida, no contexto da cultura e do sistema de valores nos quais vive e em relação aos seus objetivos,

${ }^{1}$ Enfermeira, Mestre em Enfermagem, Universidade Federal do Ceará, Fortaleza CE, Brasil (UFC); ${ }^{2}$ Enfermeira, Doutora em Enfermagem, UFC; ${ }^{3}$ Bioestatístico, Doutor em Saúde Pública, Universidade Estadual do Ceará, Fortaleza CE, Brasil (UECE).

Recebido 13 Setembro 2005, recebido na forma final 13 Dezembro 2005. Aceito 11 Fevereiro 2006.

Dra. Janaina Vall - Rua Pereira Valene 640/1902 - 60160-250 Fortaleza CE - Brasil. E-mail: janaina_vall@yahoo.com.br. 
expectativas, padrões e preocupações. Essa definição, inclui seis domínios principais: saúde física, estado psicológico, níveis de independência, relacionamento social, características ambientais e padrão espiritual ${ }^{5}$.

No Brasil, ainda são poucos os trabalhos relacionando qualidade de vida e lesão medular. No entanto avaliar a qualidade de vida é de grande importância para essas pessoas. Neste contexto, o objetivo desta pesquisa é estudar a qualidade de vida das pessoas paraplégicas, através do "The MOS 36-item Short-Form Health Survey" (SF-36), que é um instrumento genérico de avaliação, ou seja, seus conceitos não são específicos para uma determinada idade, doença ou grupo de tratamento ${ }^{6,7}$. O SF-36 é um questionário genérico multidimensional, formado por 36 itens, englobados em oito domínios: (1) capacidade funcional, (2) aspectos físicos, (3) dor, (4) estado geral de saúde, (5) vitalidade, (6) aspectos sociais, (7) aspectos emocionais e (8) saúde mental. O SF-36 re presenta o conceito de saúde que os autores acreditam ser o mais freqüentemente utilizado nas pesquisas em saúde7. Dentre os domínios descritos, os aspectos sociais são um dos mais importantes, visto que através dele é possível avaliar o contexto da qualidade de vida no quesito reinserção do portador de lesão medular à sociedade.

\section{MÉTODO}

Trata-se de uma pesquisa transversal envolvendo pacientes paraplégicos, com lesão medular completa (AIS " $A$ " segundo a $\mathrm{ASIA})^{8}$, de ambos os sexos, com idade mínima de 18 anos, que já cumpriram programa de reabilitação em uma instituição especializada e residam em Fortaleza. Os critérios de exclusão foram: tetraplégicos, não reabilitados, lesões incompletas e não-traumáticas. Segundo este perfil, obteve-se uma amostra de 32 indivíduos caracterizados na Tabela 1.

$O$ instrumento utilizado para a coleta de dados foi o questionário SF-36. Ele foi criado com trinta e seis questões que abordam, de um modo genérico, como o paciente julga sua saúde. O SF-36 foi traduzido e validado no Brasil, em tese de doutorado, por Ciconelli, em $1997^{6}$.

O questionário SF-36 foi aplicado de forma oral, na modalidade de entrevista, ou seja, as questões foram lidas em voz alta para os pacientes. Esta técnica foi preconizada ao traduzir o SF-36 para o português, devido ao baixo nível sócio-econômico e cultural dos pacientes brasileiros ${ }^{6}$. A aplicação do instrumento foi feita pela mesma pessoa para todos os pacientes, ou seja, pela própria pesquisadora, em todos os momentos.

O tratamento dos dados de qualidade de vida seguiu as orientações contidas no The SF-36 ${ }^{\circledR}$ - Health Survey Ma nual${ }^{9}$. Inicialmente foi estabelecida a correspondência de cada item ou questão à sua respectiva escala. Os dados de qualidade de vida foram então computados, considerando os valores recodificados e os escores dos itens de cada escala foram somados, os quais correspondem às respostas dos
Tabela 1. Causuística da amostra estudada.

\begin{tabular}{|c|c|c|}
\hline Características & Frequência & $\%$ \\
\hline \multicolumn{3}{|l|}{ Sexo } \\
\hline Masculino & 29 & 90,6 \\
\hline Feminino & 3 & 9,4 \\
\hline \multicolumn{3}{|l|}{ Faixa etária (anos) } \\
\hline $20-26$ & 8 & 25 \\
\hline $27-33$ & 5 & 15,6 \\
\hline $34-40$ & 7 & 21,9 \\
\hline $41-47$ & 8 & 25 \\
\hline $48-57$ & 4 & 12,5 \\
\hline \multicolumn{3}{|l|}{ Escolaridade } \\
\hline Analfabeto & 3 & 9,4 \\
\hline Ensino fundamental & 17 & 53,1 \\
\hline Ensino médio & 9 & 28,1 \\
\hline Ensino superior & 3 & 9,4 \\
\hline \multicolumn{3}{|l|}{ Ocupação } \\
\hline Sem ocupação & 16 & 50 \\
\hline Trabalha & 10 & 31,3 \\
\hline Outros $^{(1)}$ & 6 & 18,8 \\
\hline \multicolumn{3}{|l|}{ Etiologia da lesão } \\
\hline Perfuração por arma de fogo & 23 & 71,9 \\
\hline Acidente de trânsito & 4 & 12,5 \\
\hline Queda de altura ou objeto & 4 & 12,5 \\
\hline Arma branca & 1 & 3,1 \\
\hline \multicolumn{3}{|l|}{ Tempo de lesão (anos) } \\
\hline$<5$ & 11 & 34,4 \\
\hline $5-10$ & 14 & 43,8 \\
\hline $11-26$ & 7 & 21,9 \\
\hline \multicolumn{3}{|l|}{ Nivel de lesão medular } \\
\hline $\mathrm{T} 1-\mathrm{T} 4$ & 10 & 31,3 \\
\hline T5 - T9 & 12 & 37,5 \\
\hline $\mathrm{T} 10-\mathrm{T} 12$ & 10 & 31,3 \\
\hline \multicolumn{3}{|l|}{ Complicações ${ }^{(2)}$} \\
\hline Bexiga neurogênica & 30 & 93,8 \\
\hline Intestino neurogênico & 30 & 93,8 \\
\hline Espasticidade & 24 & 75 \\
\hline Dor neuropática & 18 & 56,3 \\
\hline Úlcera de pressão & 8 & 25 \\
\hline
\end{tabular}

(1)O u tros: estuda, atividades desportivas, atividades do lar; (2)O mesmo paciente pode ter mais de uma complicação.

entrevistados. Após, foi necessária a transformação de cada e s c o re numa escala de " 0 a 100 ", seguidas as orientações para somatória dos pontos e aplicado o cálculo do Raw Scale ${ }^{9}$. Esta transformação compreendeu a conversão do valor mínimo e valor máximo possível para cada item (pad ronizados segundo o manual para cálculo das escalas transformadas), em cada escala e para cada paciente. Escores entre estes valores re p resentaram a porcentagem possível de ser alcançada. Vale ressaltar que o resultado final equivale a porcentagem de qualidade de vida para cada um dos oito domínios envolvidos. Conforme aumenta a porcentagem, há melhor qualidade de vida e vice-versa.

Os resultados são expressos separadamente em domí- 
nios, visto que um valor único de qualidade de vida prop o rcionaria erros interpretativos, pois um domínio sobreporia o outro, mascarando aspectos importantes dentro do contexto amplo de avaliação da qualidade de vida. Cabe destacar também que, os dados de qualidade de vida deste estudo, re f e rem-se à percepção do paciente, como ele int e r p reta sua con dição em termos de qualidade de vida e, sabe-se que, a severidade dos sintomas e intercorrências clínicas podem alterar a qualidade de vida do portador de uma doença crônica? .

Foram respeitados os aspectos éticos concernentes a Resolução de n. 196 de 10 de outubro de 1996, que delimita d i retrizes e normas regulamentadoras de pesquisas envolvendo seres humanos ${ }^{10}$.

\section{RESULTADOS}

O resultado da avaliação da qualidade de vida das pessoas portadoras de lesão medular traumática está resumida na Tabela 2 e ilustrada no Gráfico 1. Obsena-se que as porcentagens dos domínios variaram entre 43,36 a 63,16\%, evidenciando o domínio de "aspectos sociais" como mais comprometido nos pacientes com lesão medular traumática.

\section{DISCUSSÃO}

A qualidade de vida é comprometida como um todo nas pessoas com lesão medular traumática, visto que a porcentagem máxima não ultrapassou $65 \%$. Analisando literaturas sobre o tema, são vários os estudos, embora não brasileiros, que fazem referências importantes em relação à qualidade de vida dos lesados medulares. Unalan et al. ${ }^{11}$, realizaram na Turquia um estudo com 50 pacientes sobreviventes à lesão medular morando em uma comunidade juntamente com 40 pessoas com a mesma idade sem lesão medular. Ambos os grupos preencheram o SF-36. O escore de qualidade foi significativamente baixo no grupo dos lesados medulares.

Tate et al. ${ }^{12}$, estudando mulheres com lesão medular traumática, referem baixa capacidade funcional e de bem-estar relacionados à qualidade de vida, corroborando com nossos resultados, pois a capacidade funcional ficou com uma porcentagem pouco acima de $50 \%$.

Em estudo desenvolvido nos EUA, os autores aplicaram o SF-36 em 215 pacientes com lesão medular traumática e correlacionaram principalmente os aspectos físico e mental, encontrando correlação apenas entre o componente físico e o grau de incapacidade do paciente, ou seja, quanto maior a gravidade do comprometimento decorrente da lesão medular, menor o escore do aspecto físico relacionado com a qualidade de vida $^{13}$. Em nosso estudo a porcentagem
Tabela 2. Porcentagens de qualidade de vida, segundo seus domínios, dos pacientes com lesão medular traumática.

\begin{tabular}{lcc}
\hline $\begin{array}{l}\text { Domínios da } \\
\text { qualidadede vida } \\
\mathrm{N}=32\end{array}$ & Valor obtido & $\begin{array}{c}\text { \% de qualidade } \\
\text { de vida }\end{array}$ \\
\hline Capacidade funcional & 20,75 & $53,75 \%$ \\
Aspectos físicos & 6,28 & $57,03 \%$ \\
Dor & 8,32 & $63,16 \%$ \\
Estado geral de saúde & 15,88 & $54,41 \%$ \\
Vitalidade & 15,38 & $56,88 \%$ \\
Aspectos sociais & 5,47 & $43,36 \%$ \\
Aspectos emocionais & 4,78 & $59,38 \%$ \\
Saúde mental & 20,50 & $62,00 \%$ \\
\hline
\end{tabular}

no domínio "aspectos físicos" foi $57,03 \%$, o que caracteriza um comprometimento importante no contexto da qualidade de vida.

Outroestudo desenvolvido em Atenas, na Grécia, com 38 pacientes com paraplegia, encontrou correlação entre auto-estima, satisfação com a vida e solidão, ou seja, pessoas com alta auto-estima possuem alta satisfação com a vida e menor sentimento de solidão. Ou seja, o estado mental do indivíduo, que engloba a auto-estima, está diretamente ligada ao aspecto emocional de satisfação com a vida e ao aspecto social de solidão ${ }^{14}$. Isso porque os adultos com incapacidades que reportam boa qualidade de vida construíram e mantêm um balanço entre corpo, mente e espírito, bem como harmonia com o meio ambiente $^{15}$. Em nosso estudo os domínios "aspectos emocionais" e "saúde mental" também estão comprometidos, com porcentagens de $59,38 \%$ e $62 \%$ respectivamente, demonstrando que este equilíbrio entre corpo e mente não está ocorrendo, propiciando baixa qualidade de vida, refletida em todos os seus domínios.

Da mesma forma Brillhart ${ }^{16}$ também estudou a satisfação com a vida e espiritualidade entre 230 pessoas com lesão medular de longa data. Ele concluiu que há significativa correlação positiva entre satisfação com a vida e fatores psicológicos e espirituais, de acordo com o instrumento aplicado na pesquisa. Enfatiza também o papel do enferm e i roreabilitador como suporte espiritual ao paciente, direcionandoo a redefinir suas vidas, explorando oportunidades.

Embora todos esses estudos apontem para um comprometimento global da qualidade de vida, as 


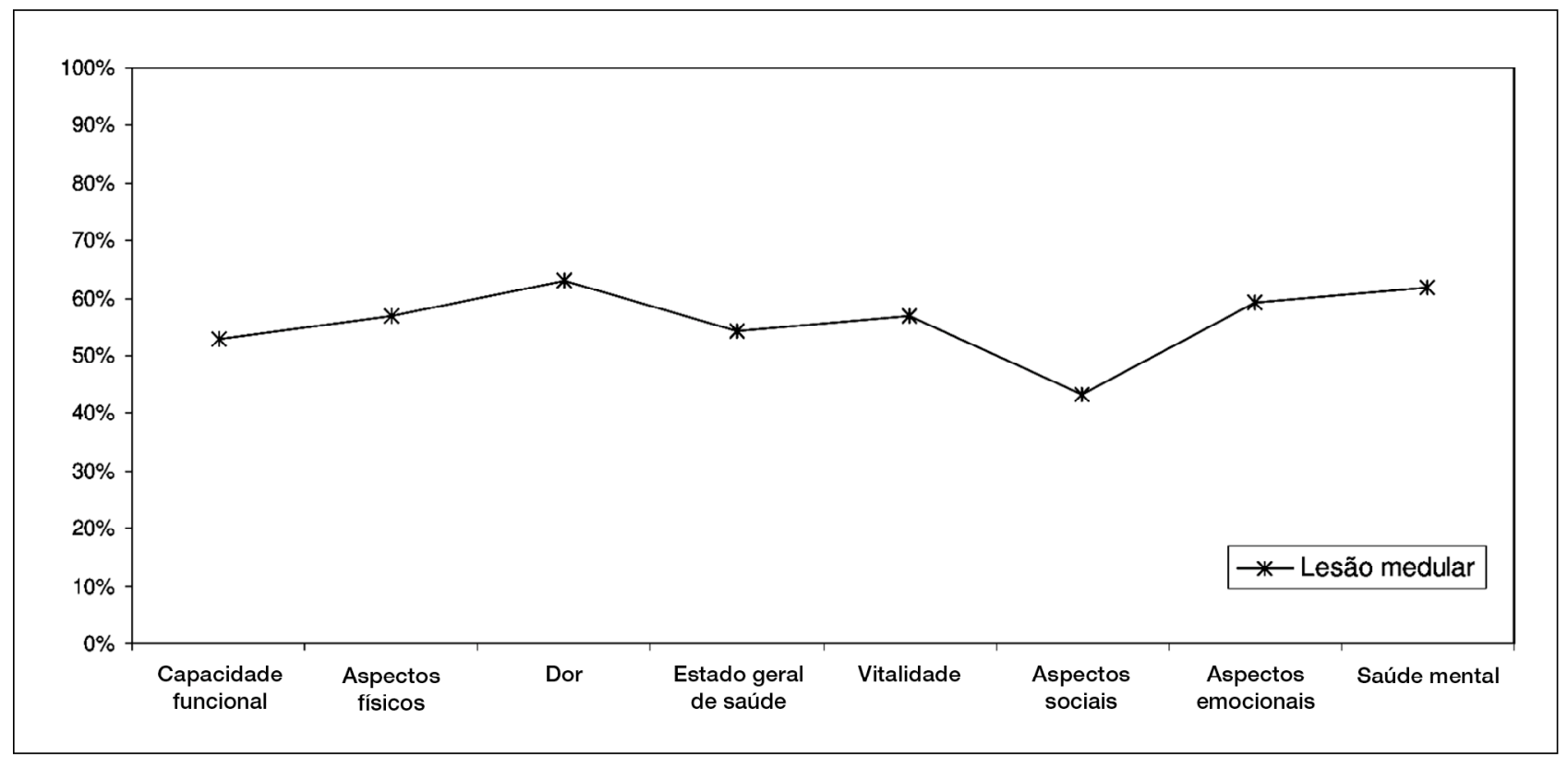

Gráfico 1. Qualidade de vida das pessoas com lesão medular traumática segundo seus domínios: capacidade funcional, aspectos físicos, dor, estado geral de saúde, vitalidade, aspectos sociais e emocionais e saúde mental.

pesquisas de Kreuter et al. ${ }^{17}$ desenvolvida na Austrália e na Suécia e Edwards et al. ${ }^{19}$, no Canadá, confirmam maior comprometimento do domínio "aspectos sociais" nos pacientes com lesão medular, corroborando os resultados do presente estudo, no qual a porcentagem de qualidade de vida neste domínio não ultrapassou $43,36 \%$, sendo o menor índice de todos os domínios avaliados.

Da mesma forma, Whiteneck et al. ${ }^{18}$ também aplicaram algumas escalas, dentre elas a de satisfação com a vida em 2726 portadores de lesão medular e obteram como resultado que fatores ambientais podem estar ligados à incapacidade, mas que esta não impede que tenham uma grande satisfação com a vida, exceto no quesito participação social. Já Hammell ${ }^{19}$ fez uma revisão de literatura explorando os conceitos de qualidade de vida ligados a lesões medulares entre o período de 1990 a 2003. A maioria dos estudos focaram pacientes com diferentes níveis de lesão medular e demonstraram, ao contrário do estudo anteriormente citado, uma insatisfação com a vida após a lesão, principalmente relacionado a uma desvantagem social.

Outroestudo que corrobora nossos resuldados é o de Johnston et al. ${ }^{20}$ que, estudando a qualidade de vida através do SF-12 entre indivíduos com lesão medular, sugerem que parte dos pacientes estudados possuem baixa escolaridade, principalmente porque, além das restrições motoras, também há restrições sociais que impedem os pacientes de seguirem com seus estudos. Leduc e Lepage, no Canadá, estudaram 587 pacientes portadores de lesão medular membro s do Quebec Paraplegic Association. Eles concluíram que pacientes mais jovens, empregados e não hospitalizados possuem melhor qualidade de vida, ou seja, esses pacientes possuem uma melhor inserção social, acarretando melhor qualidade de vida ${ }^{21}$.

Essas dificuldades e comprometimento da qualidade de vida, observados nos pacientes com lesão medular, devem-se ao fato de as modificações provocadas pela lesão ainda não poderem ser revertidas pelos recursos científicos atuais, embora já tenha pro$\mathrm{g}$ redido muito nos últimos anos ${ }^{2}$. Um estudo com 45 pacientes em condições crônicas de saúde, demonstrou que o indivíduo se depara com "novas incumbências" e problemas de ordem social, econômica e pessoal, muitas vezes de difícil enfrentamento. Além disso, demonstrou também que as alterações no estilo de vida do paciente podem ser gradual ou cumulativa, mas não ocorrem todas ao mesmo tempo ${ }^{22}$.

Os dados desta pesquisa apontam para a qualidade de vida com uma conotação individual e temporal, tendo relação com os sentimentos e percepção que o indivíduo tem de si mesmo e do mundo que o ce rca. Avaliar aspectos relativos à qualidade de vida de alguém requer que se procure captar do outro estas percepções e que se disponha de instrumentos, de modo mais fidedigno possível, que contemplem 
aspectos interelacionados e que considerem a complexidade do ser humano e a sua relação com o universo.

A avaliação da qualidade de vida é importante porque amplia as decisões da equipe de saúde, as quais se estendem aos programas e políticas assistenciais. Muitos autores são unânimes em afirmar que o fracasso de muitos programas, se deve ao fato de que eles estão ancorados na percepção dos profissionais de saúde, com intervenções desconectadas das condições sociais de qualidade de vida e, desta forma, havendo pouca investigação e valorização das reais necessidades do paciente, suas crenças e motivações ${ }^{23}$.

O SF-36, como instrumento genérico de avaliação da qualidade de vida, mostrou-se adequado à amostra estudada, podendo auxiliar não só na definição de prioridade bem como a locação de recursos de forma mais apropriada pelo sistema de saúde, além de direcionar as ações da equipe interdisciplinar de saúde, principalmente no contexto da reabilitação.

Como recomendações para novos estudos sobre o tema, sugere-se o desenvolvimento, tradução, validação e aplicação de instrumentos para avaliar a qualidade de vida desta população, sendo que esses ins$t$ rumentos devem ser validados em diferentes situações, ou seja, em diferentes centros de pesquisa e por diferentes pesquisadores em diversas populações. Também, recomenda-se que se desenvolvam mais estudos, com amostras maiores e em culturas diferentes, envolvendo não só pacientes paraplégicos, mas tetraplégicos com lesões não traumáticas. Paralelamente aos trabalhos quantitativos, também é interessante o desenvolvimento de pesquisas com enfoque qualitativo, visto que o tema é subjetivo e uma abordagem só complementa a outra. Além disso, deve haver mais envolvimento de pesquisadores e da sociedade como um todo no tema, a fim de planejar ações que vão de encontro com a melhora da qualidade de vida dos port a d o res de lesão medular, principalmente no que se refere à reinserção social, fator mais preocupante nos domínios da qualidade de vida, evidenciado nesta pesquisa.

\section{REFERÊNCIAS}

1. Rede Sarah de Hospitais de Reabilitação. Mapa da morbidade por causas externas. Disponível em www.sarah.br, acesso em 18/09/2004.

2. Salvador LA, Tarnhovi EG. Estudo comparativo da qualidade de vida em indivíduos com trauma raquimedular praticantes e não-praticantes de atividades físicas, utilizando o questionário genérico SF-36. Disponível em:http:/ / www.wgate.com.br/conteudo/medicinaesaude/fisioterapia/tr aumato/raquimedular/raquimedular.htm, acesso em 20/12/2004.

3. G reve JMD. Reabilitação na lesão da medula espinhal. Rev Med 1999; 78:276-286.

4. Andrade ACC Filho. Dor: diagnóstico e tratamento. São Paulo: Roca, 2001.

5. Dantas RAS, Sawada NO, Malerbo MB. Pesquisas sobre qualidade de vida: revisão da produção cientifica das universidades públicas do estado de São Paulo. Rev. Latino-am Enfermagem 2003;11:532-538.

6. Ciconelli RM. Tradução para a língua portuguesa e validação do questionário genérico de avaliação de qualidade de vida SF-36 (Brasil SF-36). Rev Bras Reumatol 1999;39:144-150.

7. Ware JE, Sherbourne CD. Short-form health survey (SF-36). Med Care 1992;30:473-483.

8. American Spinal Injury Association International (ASIA); Medical Society of Paraplegia. International standards for neurological classification of spinal cord injury, 2002.

9. Ware JE Júnior. SF-36 ${ }^{\circledast}$ health survey manual. Boston, Massachusets, 1993.

10. Ministério da Saúde (BR). Conselho Nacional de Saúde. Resolução n. 196 de 10 de outubro de 1996. Diretrizes e normas regulamentadoras de pesquisa em seres humanos. Mundo Saúde 1996;21:52-61.

11. Unalan H. Quality of life of primary caregivers of spinal cord injury suvivors living in the community: controlled study with short form36 questionnaire. Spinal Cord 2001;396:318-322.

12. Tate DG, Riley BB, Perna R, Roller S. Quality of life issues among women with physical disabilities or breast cancer. A rch Phys Med Rehabil 1997;78:(suppl 5):S18-S25.

13. Forchheimer M, McAweeney M, Tate DG. Use of the SF-36 among persons with spinal cord injury. Am J Phys Med Rehabil. 2004:83:390-395.

14. Tzonichaki L, Kleftaras G. Paraplegia from spinal cord injury: selfesteem, loneliness and life satisfaction. OTJR: occupation participation \& health 2002;223:96-103.

15. Atkin K. Adults with disabilities who reported excellent or good quality of life had established a balance of body, mind and spirit. Evid Based Nurs 2000;3:31.

16. Brillhart B. A study of spirituality and life satisfaction among persons with spinal cord injury. Rehabil Nurs 2005;30:31-34.

17. Kreuter M, Siosteen A, Erkholm B, Bystrom U, Brown DJ. Health and quality of life of persons with spinal cord lesion in Australia and Sweden. Spinal Cord 2005;43:123-129.

18. Whiteneck G, Meade MA, Dijkers M, Tate DG, Bushnik T, Forchheimer MB. Environmental factors and their role in participation and life satisfaction after spinal cord injury. Arch Phys Med Rehabil 2004; 85:1793-1803.

19. Hammell KW. Exploring quality of life following high spinal cord injury: a review and critique. Spinal Cord 2004;42:491-502.

20. Johnston MV, Diab ME, Kim SS, Kirshblum S. Health literacy, morbidity and quality of life among individuals with spinal cord injury. J Spinal Cord Med 2005;28:230-240.

21. Leduc BE, Lepage Y. Health-related quality of life after spinal cord injury. Disabil Rehabil 2002;24:196-202.

22. Trentini M, Silva DGV, Leimann AH. Mudanças no estilo de vida enfrentadas por pacientes em condições crônicas de saúde. Rev. Gaúch Enfermagem 1990;11:18-28.

23. Reis MG, Glashan RQ. Adultos hipertensos hospitalizados: percepção de gravidade da doença e de qualidade de vida. Rev Latino-Am Enfermagem 2001;9:51-57. 